\title{
Tully-Fisher relation, key to dark companion of baryonic matter
}

\author{
Y. Sobouti, A. Hasani Zonoozi, and H. Haghi
}

Institute for Advanced Studies in Basic Sciences (IASBS), PO Box 45195-1159, Zanjan, Iran

e-mail: [sobouti;a.hasani; haghi] @iasbs.ac.ir

Received 26 May 2009 / Accepted 20 August 2009

ABSTRACT

\begin{abstract}
Rotation curves of spiral galaxies i) fall off much less steeply than the Keplerian curves do; and ii) have asymptotic speeds almost proportional to the fourth root of the mass of the galaxy, the Tully-Fisher relation. These features alone are sufficient for assigning a dark companion to the galaxy in an unambiguous way. In regions outside a spherical system, we design a spherically symmetric spacetime to accommodate these peculiarities. Gravitation emerges in excess of what the observable matter can produce. We attribute the excess gravitation to a hypothetical, dark, perfect fluid companion to the galaxy and resort to the Tully-Fisher relation to deduce its density and pressure. The dark density turns out to be proportional to the square root of the mass of the galaxy and to fall off as $r^{-(2+\alpha)}, \alpha \ll 1$. The dark equation of state is barrotropic. For the interior of the configuration, we require the continuity of the total force field at the boundary of the system. This enables us to determine the size and the distribution of the interior dark density and pressure in terms of the structure of the observable matter. The formalism is nonlocal and nonlinear, and the density and pressure of the dark matter at any spacetime point turn out to depend on certain integrals of the baryonic matter over all or parts of the system in a nonlinear manner.
\end{abstract}

Key words. gravitation - methods: numerical - galaxies: spiral - cosmology: dark matter

\section{Introduction}

Gravitation of the observable matter in galaxies and clusters of galaxies is not sufficient for explaining their dynamics. Dark matter scenarios and/or alternative theories of gravitation (see e. g., Milgrom 1983; Behar \& Carmelli 2000; Capozziello et al. 2002, 2003, 2006; Carroll et al. 2004; Norjiri et al. 2003, 2004; Moffat 2005; Sobouti 2007) are called in to resolve the dilemma. The fact remains, however, that the proponents of dark matter have always looked for it in observable matter. No one has, so far, reported a case where there is still no baryonic matter, but there is a dynamical issue to be settled. In view of this negative observation, it has been conjectured (Sobouti 2008a,b, 2009) that, if there is a dark companion to any baryonic matter, there must be rules to connect the properties of the twin entities. On the other hand, the existence of such a rule will entitle one to interpret the case as an alternative gravity, thus reducing the difference between the two paradigms to the level of semantics. This conclusion, however, is true as long as the assumed dark matter does not interact with the baryonic one in any other way than through its gravitation.

Sobouti assumes a spherically symmetric system, attributes a dark perfect fluid companion to it, and requires the rotation curve of the system to display the same asymptotic behavior as those of the actual spirals. The reason for the assumption of a dark fluid instead of the conventionally assumed dark pressureless dust, is to ensure the satisfaction of the Bianchi identities and thereby the baryonic conservation laws (see Sect. 8 for further explanation).

In regions outside the baryonic system, he finds the density and pressure of the dark fluid companion in terms of the mass of the host system. The Tully-Fisher relation and the slow nonKeplerian decline of the rotation curves play key roles in determining the relation between the matter and its dark twin.

In this paper, we follow the same line of argument to find the structure of the dark matter in the interior of the baryonic system. The continuity of the total gravitational force at the boundary of the observable matter leads to the dark matter distribution in the interior. The Tully-Fisher relation is a nonlocal and nonlinear feature of the dynamics of galaxies: a) The presence of the total or partial integrals of the baryonic matter in the structure of both exterior and interior solutions reflects the nonlocality; b) That the excess gravitation does not increase proportionally upon increasing the mass of the host galaxy indicates the nonlinearity. To emphasize these two features, we refer to the formalism developed here as the nonlocal and nonlinear $(\mathrm{NN})$ one.

To check its validity, the formalism is applied to NGC 2903 and NGC 1560, two examples of high and low surface brightness galaxies, respectively, and the resulting rotation curves are compared with those obtained through other approaches.

\section{Model and formalism}

The following is a brief background from Sobouti (2008a,b, 2009). The physical system is a spherically symmetric baryonic matter of finite extent. By conjecture there is a dark presence that pervades both the interior and exterior of the system. The spacetime metric inside and outside of the system is necessarily spherically symmetric and takes the form

$\mathrm{d} s^{2}=-B(r) \mathrm{d} t^{2}+A(r) \mathrm{d} r^{2}+r^{2}\left(\mathrm{~d} \theta^{2}+\sin ^{2} \theta \mathrm{d} \varphi^{2}\right)$.

Let both the baryonic matter and its dark companion be perfect fluids of densities $\rho, \rho_{\mathrm{d}}$, of pressures $p, p_{\mathrm{d}}$, respectively, and be at rest. From the field equations of general relativity (GR), we find

$\frac{1}{r^{2}}\left[\frac{\mathrm{d}}{\mathrm{d} r}\left(\frac{r}{A}\right)-1\right]=-\left(\rho+\rho_{\mathrm{d}}\right)$

$\frac{1}{r A}\left(\frac{B^{\prime}}{B}+\frac{A^{\prime}}{A}\right)=\left[\left(p+p_{\mathrm{d}}\right)+\left(\rho+\rho_{\mathrm{d}}\right)\right]$,

where we have let $8 \pi G=c^{2}=1$, and " $=\mathrm{d} / \mathrm{d} r$. In the nonrelativistic regime, we neglect the pressures, eliminate the densities 
between the two equations, and arrive at

$\frac{B^{\prime}}{B}=\frac{1}{r}(A-1)$.

In the following two sections we solve Eqs. (2)-(3) inside and outside the baryonic system.

\section{Exterior solution}

Hereafter, the parameters pertaining to the interior and exterior of the system will be labeled by the superscripts $(i)$ and $(e)$, respectively. The unknowns in Eqs. ((2)-(3)) are $A, B, \rho_{\mathrm{d}}, p_{\mathrm{d}}$, and the dark equation of state. We begin with Eq. (3) and assume that in the baryonic vacuum, $\rho=p=0$, the factor $\left(A^{(e)}-1\right)$ is differentiable and has the series expansion

$\left(A^{(e)}-1\right)=\left(\frac{r_{0}}{r}\right)^{\alpha}\left(s_{0}+\frac{s_{1}}{r}+\cdots\right), \quad r \geq R$,

where the indicial exponent $\alpha$ and $s_{0}$ are dimensionless, $s_{1}$ has the dimension of length, $r_{0}$ is an arbitrary length scale of the system, and $R$ is the radius of the baryonic sphere. Substituting Eq. (4) into Eq. (3) and integrating the resulting expression, gives

$B^{(e)}=\exp \left[-\left(\frac{r_{0}}{r}\right)^{\alpha}\left(\frac{s_{0}}{\alpha}+\frac{s_{1}}{(1+\alpha) r}+\cdots\right)\right]$.

We expand the exponential factor, keep its first two terms, and for the weak field gravitational potential, $\phi=(B-1) / 2$, find

$\phi^{(e)}=-\frac{1}{2}\left(\frac{r_{0}}{r}\right)^{\alpha}\left[\frac{s_{0}}{\alpha}+\frac{s_{1}}{(1+\alpha) r}+\cdots\right]$.

The square of the circular speed of a test object orbiting the galaxy is

$v^{2}=r \frac{\mathrm{d} \phi^{(e)}}{\mathrm{d} r}=\frac{1}{2}\left(\frac{r_{0}}{r}\right)^{\alpha}\left(s_{0}+\frac{s_{1}}{r}+\cdots\right)$.

Equation (7) is the rotation curve of our hypothetical galaxy in its baryonic vacuum. It has an asymptotically constant logarithmic slope,

$\Delta=d \ln v^{2} / d \ln r \rightarrow-\alpha$ as $r \rightarrow \infty$.

\subsection{Determination of $\alpha, s_{0}, s_{1}, \cdots$}

Rotation curves of actual spiral galaxies have two distinct nonclassical features:

i) Their asymptotic slopes are much flatter than that of the Keplerian curves, -1, (Sanders 1996; Bosma 1981; Begmann 1989; Persic \& Salucci 1995; Begmann et al. 1991; Sanders \& Verheijen 1998; Sanders \& McGhaugh 2002). This implies $\alpha \ll 1$. From Persic et al. 1996, who study 1100 galaxies with the aim of arriving at a universal rotation curve, we estimate

$\alpha<0.01$.

Moreover, $\alpha$ does not seem to be a universal constant. The rotation curves of more massive galaxies appear to fall off somewhat more steeply than those of the less massive ones (Persic et al. 1996). Hereafter, for simplicity but mainly for pedagogical reasons, we work in the limit of $\alpha \rightarrow 0$. ii) Their asymptotic speeds follow the Tully-Fisher relation. They are almost proportional to the fourth root of the mass of the host galaxy (Tully \& Fisher 1977; Begmann 1989; McGaugh et al. 2000; McGhaugh 2005). In Eq. (7), letting $\alpha \rightarrow 0$, the dominant term at large distances is $v^{2}=s_{0} / 2$. We identify this $v$ with the Tully-Fisher asymptote and conclude that

$$
s_{0}=\lambda\left(M / M_{\odot}\right)^{1 / 2}, \lambda=2.8 \times 10^{-12} \text {, }
$$

where $M$ is the galactic mass, and $\lambda$ can be obtained either from a direct examination of the observed asymptotic speeds (Sobouti 2007) or from a comparison of the first term of Eq. (7) with the low acceleration limit of MOND (Milgrom 1983): $v^{2} / r \rightarrow\left(a_{0} g_{N}\right)^{1 / 2}, a_{0}=1.2 \times 10^{-10} \mathrm{~m} \mathrm{~s}^{-2}$ (Begmann 1989).

Again letting $\alpha \rightarrow 0$, the second term in Eq. (7) is the classic Newtonian or GR term. Therefore, $s_{1}$ should be identified with the Schwarzschild radius of the host galaxy:

$s_{1}=2 G M / c^{2}$.

Here, for clarity, we have restored the constants $c^{2}$ and $G$ and written $s_{1}$ in physical units. There is no compelling observational evidence to indicate the need for other terms in Eqs. (4)-(7). Therefore, at least at the present state of the extent and accuracy of the observational data, we truncate the series at the $s_{1}$ term.

\section{Interior solution}

The first and foremost condition to be satisfied is the continuity of the total force exerted on a test object at the boundary, $R$, of the baryonic system. Pressure forces are anticipated to be insignificant in the present problem so are ignored. These gravitational forces remain. From Eqs. (7)-(10), the exterior force is

$\frac{\mathrm{d} \phi^{(e)}}{\mathrm{d} r}=\frac{1}{2}\left[\lambda\left(\frac{M}{M_{\odot}}\right)^{1 / 2} \frac{1}{r}+\frac{2 G M}{c^{2}} \frac{1}{r^{2}}\right], r \geq R$.

By analogy, for the interior of the system we adopt

$\frac{\mathrm{d} \phi^{(i)}}{\mathrm{d} r}=\frac{1}{2}\left[\lambda\left(\frac{M(r)}{M_{\odot}}\right)^{1 / 2} \frac{1}{r}+\frac{2 G M(r)}{c^{2}} \frac{1}{r^{2}}\right], r \leq R$,

where $M(r)=4 \pi \int_{0}^{r} \rho r^{2} \mathrm{~d} r$ is the variable baryonic mass inside the radius $r$. The continuity of the exterior and interior forces at the boundary is evident, QED. Once the baryonic $\rho(r)$ and $M(r)$ are known, $\phi^{(i)}(r), B^{i}(r) \approx 1+2 \phi^{(i)}$ and $A^{(i)}$ can be integrated. The expression for the latter is much simpler and is given below for later reference. From Eq. (3) we find

$A^{(i)}-1=2 r \frac{\mathrm{d} \phi^{(i)}}{\mathrm{d} r}=\left[\lambda\left(\frac{M(r)}{M_{\odot}}\right)^{1 / 2}+\frac{2 G M(r)}{c^{2}} \frac{1}{r}\right]$.

This has the same form as Eq. (4), where $M$ is replaced by $M(r)$.

\section{Structure of the dark matter}

The densities are obtained from Eq. (2) or equivalently from Poisson's equation through Eqs. (11)-(12). For the exterior dark density we find

$\rho_{\mathrm{d}}^{(e)}(r)=\lambda\left(\frac{M}{M_{\odot}}\right)^{1 / 2} \frac{1}{r^{2}}, \quad r \geq R$. 
Note the square root dependence on the mass of the galaxy and the fall out as $r^{-2}$. For the interior, $A^{(i)}$ is given by Eq. (13), whose first term gives the interior dark density and the second renders the baryonic density, $\rho$. Thus,

$\rho_{\mathrm{d}}^{(i)}(r)=\lambda\left[\frac{M(r)}{M_{\odot}}\right]^{1 / 2} \frac{1}{r^{2}}\left[1+2 \pi \frac{\rho r^{3}}{M(r)}\right], \quad r \leq R$.

The dark matter inside the radius $r$ is

$M_{\mathrm{d}}(r)=4 \pi \int_{0}^{r} \rho_{\mathrm{d}}(r) r^{2} \mathrm{~d} r=\lambda\left[\frac{M(r)}{M_{\odot}}\right]^{1 / 2} r$.

Equation (16) holds for any $r$. For $r \geq R$, however, $M(r)$ attains its maximum constant value, $M$, and $M_{\mathrm{d}}(r>R)$ becomes proportional to $r$.

It is instructive to look at the behavior of Eq. (15) in the neighborhood of the origin, where $\rho \rightarrow \rho_{c}$ and $M(r) \rightarrow$ $4 \pi \rho_{c} r^{3} / 3$. Equation (15) tends toward

$\rho_{\mathrm{d}}^{(i)}(r \rightarrow 0)=\frac{5}{2} \lambda\left(\frac{4 \pi}{3} \frac{\rho_{c}}{M \odot}\right)^{1 / 2} r^{-1 / 2}$.

Similarly,

$M_{\mathrm{d}}^{(i)}(r \rightarrow 0)=\lambda\left(\frac{4 \pi}{3} \frac{\rho_{c}}{M_{\odot}}\right)^{1 / 2} r^{5 / 2}$.

While the density becomes singular as $r \rightarrow 0$, no cusp develops. For the measure $r^{2} \mathrm{~d} r$ tends to zero as $r \rightarrow 0$.

Pressures of the matter and of its dark companion are obtained from their hydrostatic equilibrium, a requirement of the Bianchi identities. The general formula is

$\frac{p^{\prime}}{p+\rho} \approx \frac{p^{\prime}}{\rho}=-\frac{1}{2} \frac{B^{\prime}}{B} \approx-\frac{\mathrm{d} \phi}{\mathrm{d} r}$.

For the exterior pressure from Eqs. (19), (14), (11), we find

$p_{\mathrm{d}}^{(e)}(r)=\frac{1}{4} s_{0}\left(\frac{s_{0}}{r^{2}}+\frac{2}{3} \frac{s_{1}}{r^{3}}\right), \quad r \geq R$.

The presence of an extra factor of $s_{0}$ in Eq. (20) makes the pressure an order of magnitude less than the density and justifies the approximation made in the derivation of Eq. (3) and thereafter. The equation of state, $p(\rho)$, in the exterior region is obtained by eliminating $r$ between Eqs. (20) and (14). It is barrotropic. The internal pressure is obtained in a similar way. It is, however, too involved expression to give here.

A pedagogical note: Throughout the text, except in Eq. (10), we have chosen $8 \pi G=c^{2}=1$. To write the results in physical units, the rule is to multiply, everywhere, the potentials, $\phi$, by $c^{2}$, the dark densities, $\rho_{\mathrm{d}}$, dark masses, $M_{\mathrm{d}}$, by $c^{2} / 8 \pi G$, and the dark pressures, $p_{\mathrm{d}}$, by $c^{4} / 8 \pi G$.

\section{Application to actual spirals}

Spiral galaxies are flattened objects. Their approximation as spherical systems introduces an error on the order of $\left(R_{\mathrm{gyr}} / r\right)^{2}$, where $R_{\mathrm{gyr}}(r)$ is the gyration radius of the mass enclosed within a radius $r$. In a flat system that thins out as an exponential or as a Kuzmin disk, say, this ratio would be a few parts in thousand and small enough for our purpose. This is also the practice of all the authors quoted so far in this paper. To illustrate the practical applicability of the formalism developed here, we construct the rotation curves of two standard high- and low-surface brightness
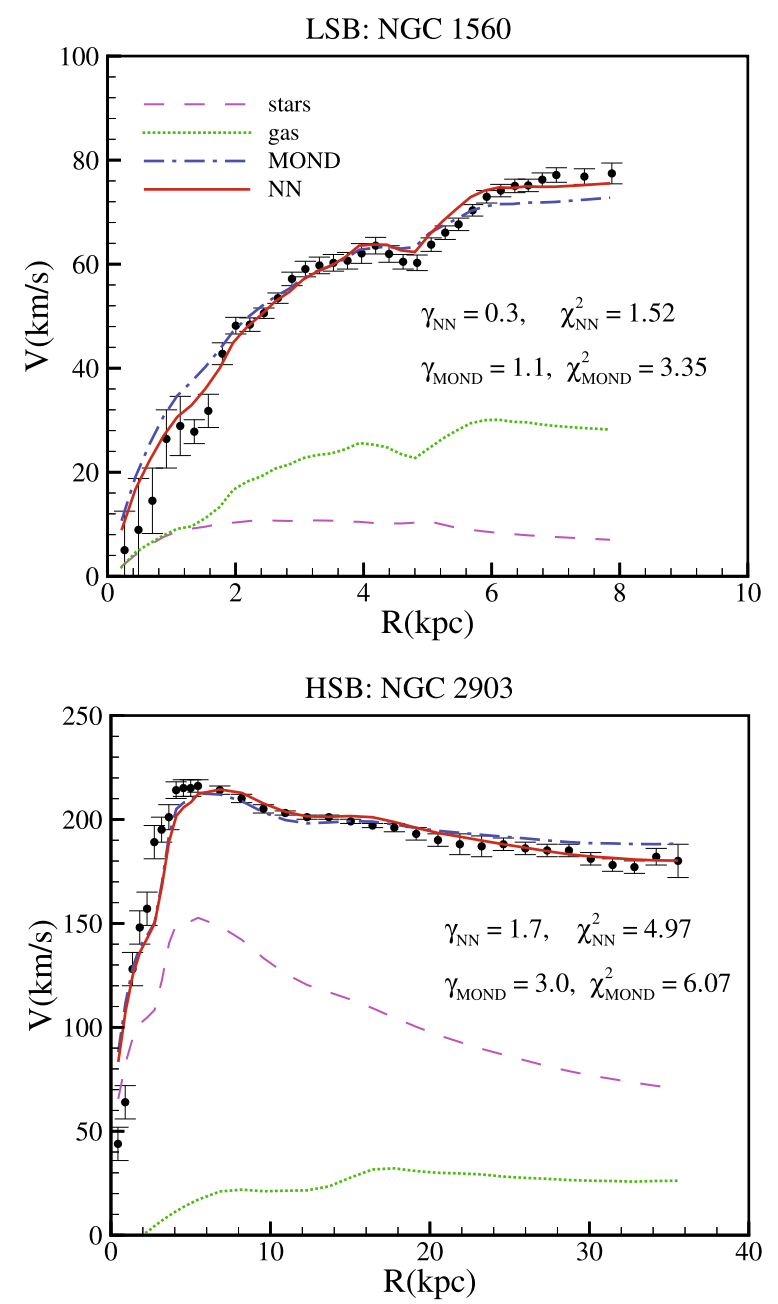

Fig. 1. Points with error bars are observed data. Dotted and dashed lines are the contributions of the gaseous and stellar components to the rotation curves, respectively. Dashed-dotted line is the rotation curve constructed through MOND's formalism. Solid line is our rotation curve calculated from Eq. (12). The free parameter in matching theoretical curves to data points, is the stellar mass-to-light ratio.

galaxies and compare the results with those obtained through MOND's formalism.

NGC 2903 is a textbook example of a high surface brightness spiral. It has a large stellar component and small HI content. The gas is confined to the galactic plane and follows circular orbits. It is well observed out to about $40 \mathrm{kpc}$ (Begmann 1989). In contrast, NGC 1560 is a low surface brightness spiral with a dominant gas component. Its observed rotation curve extends out to about $8 \mathrm{kpc}$ and does not seem to have reached its asymptotic regime.

In Fig. 1 we construct the rotation curves of our NN formalism from Eq. (12), in which $M(r)$ is the total, stellar plus HI, mass interior to $r$. The free adjustable parameter in matching the theoretical curves to data points, is the "stellar" mass-to-light ratio, $\Upsilon$, assumed to be constant throughout the galaxy. For comparison we have also included the rotation curves of MOND. That the NN curves trace the data points more closely than the MOND ones can be seen pictorially. The $\chi^{2}$ test and $\Upsilon$ 's of Table 1 , however, illustrate this in a quantitative way. In both galaxies our $\chi_{\mathrm{NN}}^{2}$ is noticeably small. Significant, however, is the low stellar mass-to-light ratio of the young and gas-dominated NGC 1560 . Our $\Upsilon=0.3$ is, by far, closer to 0.4 estimate of McGaugh (2002) than to 1.1 of MOND. 
Table 1. Minimum $\chi^{2}$ and fitted stellar mass-to-light ratio, $\Upsilon$, of MOND and of our NN formalism.

\begin{tabular}{ccccc}
\hline \hline Galaxy & $\chi_{\mathrm{NN}}^{2}$ & $\Upsilon_{\mathrm{NN}}$ & $\chi_{\mathrm{MOND}}^{2}$ & $\Upsilon_{\mathrm{MOND}}$ \\
\hline NGC 2903 & 4.97 & 1.7 & 6.07 & 3.0 \\
NGC 1560 & 1.52 & 0.3 & 3.35 & 1.1 \\
\hline
\end{tabular}

Our next project is to study pressure-supported systems, globular clusters and dwarf spheroidal galaxies (dSph). Globular clusters are commonly believed to be almost Newtonian systems, while dSph's show significant deviations from Newtonian regimes. The low baryonic mass and extremely high dynamicalmass-to-light ratio of dSph's are inconsistent with population synthesis models (Hilker 2006; Jordi et al. 2009; Angus 2008). Our approach is to find a counterpart of the classical virial theorem for our proposed gravity and to solve a modified Jeans equation. The aim is to verify whether the velocity dispersions obtained via Jeans equation fit the observed data. We also hope to be able to come up with a notion equivalent to the fundamental plane for galaxies where one arranges the galaxies on a two-parameter-plane in a three-dimensional space of luminosity, velocity dispersion, and some other global characteristics of the galaxies.

\section{Nonlocality and nonlinearity of the formalism}

The masses $M$ and $M(r)$ are integrals over all or parts of the system. Their presence, in the structure of the spacetime metric, in the rotation curve, in the expressions for the dark densities and pressures, etc., reflects the nonlocal nature of the theory. That these integrals enter the formalism not in a linear way indicates the nonlinearity of it. Both features are rooted in the Tully-Fisher relation, which requires the dynamical variables at one spacetime point to depend on the integral properties of the whole or parts of the system through the square root of these integrals. Any attempt to derive the spacetime metric entertained in this paper through a variational principle should take these two features into account.

In this respect, Hehl and Mashhoon's generalization of GR, (Hehl \& Mashhoon 2009a,b), constructed within the framework of the translational gauge theory of gravity, is interesting. In the weak field approximation, the excess gravitation coming from the nonlocality of their theory can be interpreted as a dark companion to the baryonic matter. In the case of a point baryonic mass, $M$, the dark density has the expected $r^{-2}$ distribution, But it does not obey the Tully-Fisher relation. Instead of $M^{1 / 2}$, it is proportional to $M$ itself.

\section{Concluding remarks}

The formalism developed here is a dark matter scenario or, equivalently, a modified GR paradigm to understand the nonclassical behavior of the rotation curves of spiral galaxies. Following (Sobouti 2008a,b, 2009), we attribute a hypothetical dark perfect fluid companion to our model galaxy, and find the size and the distribution of the companion by comparing the rotation curve of the model with those of the actual galaxies. However, as long as the dark companion displays no physical characteristics other than its gravitation, one has the option to interpret the scenario as an alternative theory of gravitation. Here, for example, one may maintain that the gravitation of a baryonic sphere is not what Newton or Schwarzschild profess, but rather what one infers from the spacetime metric detailed above. In fact we wish to emphasize that any modified gravity is expressible in terms of a dark matter scenario. And vice versa, any dark matter paradigm, in which the matter and its dark twin are related by certain rules, is explainable by a modified gravity. The difference between the two alternatives is semantic.

Dynamics of galaxies is a nonrelativistic issue. Yet, its analysis in a GR context answers questions that otherwise are left out. In particular, in a nonrelativistic scenario, there is neither need nor logic to assign a pressure field to a hypothetical matter that one knows nothing about its nature. In a GR context, on the other hand, the dark matter has to have a pressure field and has to be in hydrodynamic equilibrium as a requirement of the Bianchi identities and thereby of the conservation laws of the baryonic matter, i.e. the vanishing of the 4-divergence of both sides of the field equations. Let us also note in passing that all those metric approaches that attempt to explain the galaxy problems with the aid of a single scalar field are subject to the same criticism, namely the violation of the Bianchi identities and of the conservation laws.

Regions outside to the baryonic matter are not dark matter vacua. Therefore, the Ricci scalar does not vanish, and there are excess lensing and excess periastron precession caused by the dark matter. These are analyzed in Sobouti (2008a,b, 2009).

The formalism is good for spherical distributions of baryonic matters. An axiomatic generalization to nonspherical configurations or to many body systems requires further deliberations and more accurate observational data to help find some solutions. One might need other postulates not contemplated. The difficulty lies in the nonlinearity of the formalism. There is no superposition principle. One may not add the fields of the dark companions of two separate baryonic systems because $s_{0}$ of Eq. (9) is not linear in $M$ or $M(r)$.

Acknowledgements. We thank S. S. McGaugh for providing us with observational data on the rotation of galaxies and for his useful comments on their interpretation.

\section{References}

Angus, G. W. 2008, MNRAS, 387, 1481

Bosma, A. 1981, AJ, 86, 1825

Begmann, K. G. 1989, A\&A, 233, 47

Begmann, K. G., Broeils, A. H., \& Sanders, R. H. 1991, MNRAS, 249, 523

Behar, S., \& Carmeli, M. 2000, Int. J. Theor. Phys., 39, 1397

Capozziello, S. 2002, Int. J. Mod. Phys. D., 11, 483

Capozziello, S., Cardone, V., Carloni, S., \& Troisi, A. 2003, Int. J. Mod. Phys. D., 12, 1969

Capozziello, S., Cardone, V. F., \& Troisi, A. 2006, JCAP, 8, 1

Carroll, S., Duvvuri, V., Trodden, M., \& Turner, M. 2004, Phys. Rev. D, 70, 2839

Hehl, F. W., \& Mashhoon, B. 2009a, Phys. Lett. B, 673, 279

Hehl, F. W., \& Mashhoon, B. 2009b, Phys. Rev. D, 79, 064028

Hilker, M. 2006, A\&A, 448, 171

Jordi, K., Grebel, E. K., Hilker, M., et al. 2009, AJ, 137, 4586

McGhaugh, S. S. 2005, ApJ, 632, 859

McGhaugh, S. S., Schombert, J. M., Bothun, G. D., \& de Blok, W. J. G. 2000, ApJ, 533, L99

Milgrom, M. 1983, ApJ, 270, 365, 371, 384

Moffat, J. W. 2005, JCAP, 2005, 003

Nojiri, S., \& Odintsov, S. D. 2003, Phys. Rev. D, 68, 123512

Nojiri, S., \& Odintsov, S. D. 2004, General Relativity and Gravitation, 36, 1765

Persic, M., Salucci, P., \& Stel, F. 1996, MNRAS, 281, 27

Persic, M., \& Salucci, P. 1995, ApJS, 99, 501

Sanders, R. H. 1996, ApJ, 473, 117

Sanders, R. H., \& McGhaugh, S. S. 2002, ARA\&A, 40, 263

Sanders, R. H., \& Verheijen, M. A. W. 1998, ApJ, 503, 97

Sobouti, Y. 2007, A\&A, 464, 921

Sobouti, Y. 2008a, [arXiv: 0810.2198]

Sobouti, Y. 2008b, [arXiv:0812.4127v2]

Sobouti, Y. 2009, [arXiv: 0903.5007]

Tully, R. B., \& Fisher, J. R. 1977, A\&A, 54, 661 